\title{
General Identification and Solutions of Computer Network Fault
}

\author{
Yuanyuan Chen \\ Nanchang Institute of Science \& Technology
}

Keywords: Computer; Network failure; Fault analysis; Solution

\begin{abstract}
Currently, the network informatization develops rapidly. With living standard rising, computer usage rate is higher and the resulting problems also become more. Computer network failure is inevitable, so the solution of network fault is one of the important works of network management. We must carefully study the technical theory of computer network; so that people can get the fastest speed to restore the operation of network system, ensuring the form of network smooth. This paper mainly discusses the issues of computer network fault and general recognition of common faults in the network, and provides corresponding solutions for different common network fault.
\end{abstract}

\section{Introduction}

Currently, computer network technology changes with each passing day. In the era of rapid development, computer network extends every corner of the world and is used in all walks of life, spreading to every family. It has brought a lot of convenience to people, but also brought a lot of troubles at the same time. The combination of computer technology and network technology makes people's production, living and learning modes have very big change, in which computer technology is the extension and expansion of network technology. Computer network has enriched the life of people, making work, study convenient and efficient. However, with the development of computer network, the influence of network fault forces people to focus on them. Network fault is very common and various and it needs timely analysis and maintenance, with the fastest speed to restore the normal operation of network. It is the key to master a set of effective network maintenance theory, method and technology.

The author introduces the common network fault classification and screening methods, and according to the common network fault classification as: physical fault and logic fault, then the solutions for each fault are discussed.

\section{Basic Concepts of Computer Network}

Computer network is a system integrating computer, and composed by the means of communication facilities. Briefly, it is to concentrate the computers around the world together through various means of communication in order to meet the mutual communication, mutual connection and sharing of all kinds of hardware and software resource. LAN and WAN are the two forms that are usually defined as computer network. The characteristic of local area network (LAN) is small coverage area, and it generally covers one or two buildings. WAN is characterized by large coverage area, such as between companies, countries and even the whole world. Due to the rapid development of computer network, it is bound to cause various forms of network connection. There is a way that is very easy to expand the Internet, namely using a unified agreement to complete different network interconnection. Internet is a good example, and it just uses this way to realize the connection of networks.

\section{Computer Network Fault Identification}

Physical Fault. Physical fault is hardware failure and the form for this kind of failure is diverse. It is also one of the more common faults for network equipment and relatively easy to solve. The most common form of network is unstable connection, sometimes even complete disconnected. 
Port Fault. Port fault mainly has two cases: unstable port and port failure. Screening methods: port usage conditions will be reflected to the other devices signal light. As a result, fault area and the reason can be determined by observing the status of these signal lights. In addition, other ports can be used for testing. If communication is normal, which means there is a problem for it; if the communication is failed, port fault is ruled out.

Switches or Routers Breakdown. Switch or router fault refers to equipment damage, resulting in abnormal work and network fault. Screening methods: determine whether the switch or router equipment in good condition, and there are two simple ways: first, the method of observation. Observe indicator light, in normal condition, switches and routers light should be green; otherwise, the equipment can be judged as damaged. Second is replacement method. The Internet and computer in normal use can be used to connect devices, if the communication restore to normal, it means the equipment is normal; if the communication is still failed, the port of switch or router should be screened.

Network Card Fault. Network card fault belongs to host hardware failure. As network terminal, network card failure is also an important cause of network failure. As the network card is usually installed within the host, its fault has certain concealment. In addition, the network adapter failure also has diverse forms, such as loose network card, network card hardware failure, host card slot fault and so on, which can lead to abnormal work of network card.

Logic Fault. Logical failure is software failure which is corresponding to the physical fault. When computer has logic problems, the network speed is quick and slow sometimes, bringing adverse effects to web browsing. Physical fault identification and screening is "visible and tangible", and therefore is relatively simple. Logical fault cause is relatively complex, so the difficulty for screening is higher. These kinds of failures often need to rely on specialized technical personnel to help solve. It mainly includes the host logical fault, wrong host network address parameter setting, improper host network protocols or services installation, improper network card driver installation, some important process or port closed, and so on.

\section{Some Common Problems and Solutions of Network Fault}

(1) Fault Phenomenon: the Network Adapter Set and Computer Resources. Analysis, elimination: avoid conflict with other computer resources by adjusting the IRQ and I/O values. In some cases, the motherboard jump line also needs to adjust to prevent the conflicts with other resources.

(2) Fault phenomenon: the clients in the local area network (LAN) of Internet cafes can see each other on the "network neighborhood" online, and only a computer the others can't see, it also can't see the other computer. (The premise: the Internet cafes LAN is connected via a HUB or switch into a star network structure).

Analysis and troubleshooting: check if the computer system work is normal; check the network configuration of the computer; check the computer's network card is working correctly; check if there is conflict for the network adapter settings in this computer and other resources; check whether the cable is disconnected; check whether the cable connector contact is normal.

(3) Fault phenomenon: the local area network (LAN) of Internet cafes has two segments; all computers in one of the segments cannot connect the Internet. (The premise: the Internet cafes LAN connects to the network segment through two HUB or switches).

Analysis, troubleshooting: the main trunk line of two network segments is broken or the joints are bad. Check the settings for the network segment.

(4) Fault phenomenon: all the clients in the local area network (LAN) of Internet cafes can see each other on the "network neighborhood" online. (The premise: the Internet cafes LAN is connected via a HUB or switch into a star network structure).

Analysis and troubleshooting: check whether the HUB or switch works normally.

(5) Fault phenomenon: a client computer in the local area network (LAN) of Internet cafes can see server, but cannot connect the Internet. (The premise: the server refers to the computer that other client computers connect Internet cafes through it, the same below) 
Analysis and troubleshooting: check the client TCP/IP Settings, check the IE browser settings in the client machine, and check the settings for the client machine in server.

(6) Fault phenomenon: for dial-up operation, MODEN has no voice of dialing, and the Internet cannot be connected, and the MODEN lights don't flash.

Analysis, troubleshooting: check if the telephone line is busy at the moment; if the connection of MODEN server (including: wire, joint) is normal; if the line is normal, if having any noise interference; if dial-up network configuration is correct; if MODEN configuration settings is correct; check if the dial tone audio or pulse mode is normal.

\section{Conclusion}

Nowadays, the combination of computer and network technology has changed people's life and learning mode. As the computer network technology is developing rapidly and network fault is very complex, all kinds of network fault emerge in endlessly. Computer network has very high speed, low production cost, so it is quickly accepted by people, and become one of the important ways that people process daily office data. The resulting network faults are becoming more and more common and have various types, and we must have a professional knowledge of network theory foundation and practical skills of network maintenance that to deal with network failure problems timely, and make network maintenance quick and effective so that the network can restore normal with the fastest speed. This paper summarizes the common categories of fault and screening methods. For specific diagnostic techniques, on the network fault diagnosis, the general principle for dealing with the network fault is "software first, hardware second", but in practice, it will depend on specific analysis, to find out the corresponding solutions.

\section{References}

[1] Qader K. Computer Network Faults Classification Using Hybrid (FCM and PNN) Technique[C]// Faculty of Technology Conference- Portsmouth University. 2014.

[2] Zhu C C. Research on Computer Network Fault Diagnose Based on Support Vector Machines [J]. Computer Simulation, 2011, 28(10):103-106.

[3] Wang Q. Computer Network Fault Diagnosis Based On Neural Network [J]. International Journal of Future Generation Communication \& Networking, 2015, 8.

[4] Popescu D E, Lonea M. Some Aspects about Vagueness \& Imprecision in Computer Network Fault-Tree Analysis [J]. International Journal of Computers Communications \& Control, 2010, V (4):558-566.

[5] Zhong J. Analyses the Causes and Countermeasures of Computer Network Fault [J]. Computer Knowledge \& Technology, 2013.

[6] Zhang C L. The Research of the Computer Network Fault Solution [J]. Advanced Materials Research, 2014, 971-973:1824-1827.

[7] $\mathrm{Lu} \mathrm{M}$. Research on intelligence of computer network fault management [J]. Electronic Test, 2013.

[8] Wang X W. The Computer Network Fault Analysis and Maintenance of Common [J]. Science \& Technology Information, 2011.

[9] Feng B. Higher Vocational College Computer Network Fault Analysis and Management Countermeasures [J]. Friend of Science Amateurs, 2011.

[10]Huang P. Computer Network Fault Identification and Solutions [J]. Computer Knowledge \& Technology, 2013.

[11]Zhang J X, Gang J I, Zheng Q S. Research on Rough Set Neural Network Computer Network 
Fault Diagnosia Method [J]. Modern Machinery, 2005.

[12]Ge Liang Yan Chenghua Yu Hongyue Department of the Information Security, Naval University of Engineering, Wuhan. Computer Network Fault Detection Based on Neural Network [J]. Computer \& Digital Engineering, 2010. 